\title{
Autoinoculation lupus vulgaris of the perineum
}

\author{
V N Sehgal, A K Chaudhry, R Gupta
}

\begin{abstract}
The case of a young heterosexual male, with a 7 year history of an asymptomatic progressive plaque over the right side of the perineum is described, which 4 years later involved the left perineum and scrotal skin, indicating autoinoculation. The diagnosis of lupus vulgaris was made by strongly positive tuberculin test, histopathology, and a favourable response to a short course of intensive antitubercular therapy.
\end{abstract}

\section{Introduction}

Lupus vulgaris is a well-recognised entity, ${ }^{1}$ caused by Mycobacterium tuberculosis, and is an expression of augmented cell-mediated immunity. It occurs in relatively young individuals with a high degree of tuberculin sensitivity. It is a chronic progressive disease, which may either be the result of direct extension, lymphatic or haematogenous spread. Occasionally, it may follow primary inoculation tuberculosis, BCG vaccination, and in a few cases it may be preceded by scrofuloderma. Lupus vulgaris affecting the perineum is unusual, and hence is being reported.

\section{Case report}

A 33 year old married heterosexual male presented with a 7 year history of a small, pea-sized, brownish red asymptomatic eruption on the right side of the perineum. In the absence of any specific treatment it increased in size. Four years later the patient noticed a similar eruption on the left side of the perineum and adjacent scrotal skin. There was no history of trauma, homosexual or extramarital heterosexual contact or history suggestive of tuberculous involvement of any other part of the body. The family history was not significant.

Cutaneous examination revealed two well-defined erythematous plaques with irregular margins, the surface was dry and scaly with lateral borders showing multiple brownish red, soft to firm, pea-sized swellings with a smooth shiny surface. The size of the

Department of Dermatology and Venereology, Lady Hardinge Medical College and Maulana Azad Medical College and Associated Hospitals, New Delhi

V N Sehgal, A K Chaudhry, R Gupta

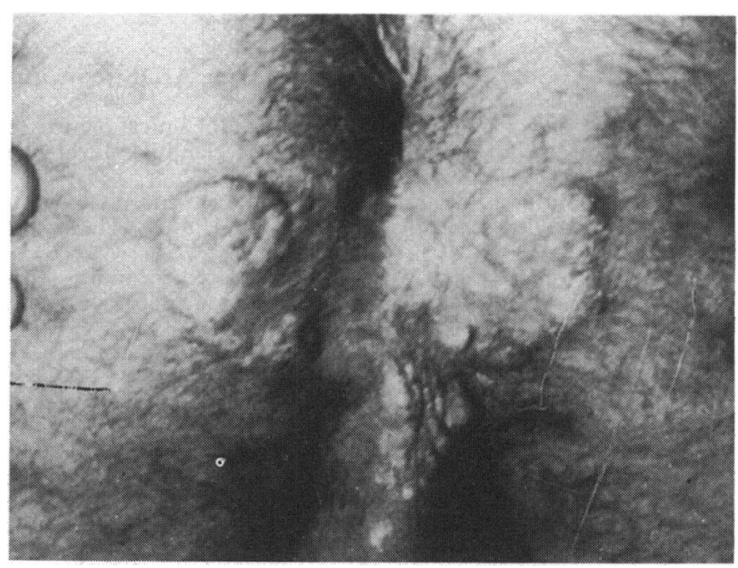

Figure 1 Erythematous plaque with irregular margin on left and right perineum, with atrophy and scarring.

lesion was $3 \times 3 \mathrm{~cm}$ on the right side of perineum and $7 \times 3 \mathrm{~cm}$ on the left and on the scrotum. Areas of atrophy and scarring were present in the centre and medial border of the lesion (fig 1). Diascopic examination revealed apple-jelly nodules. There was no clinically significant lymphadenopathy and no BCG vaccination scar on the arm.

The results of lymphocyte counts, urine analysis, liver and renal functions tests were within normal limits. The Mantoux test (with 1 TU of purified protein derivative) read $25 \mathrm{~mm} \times 22 \mathrm{~mm}$. The ESR was $23 \mathrm{~mm}$ (1st h) by Westergren method. The

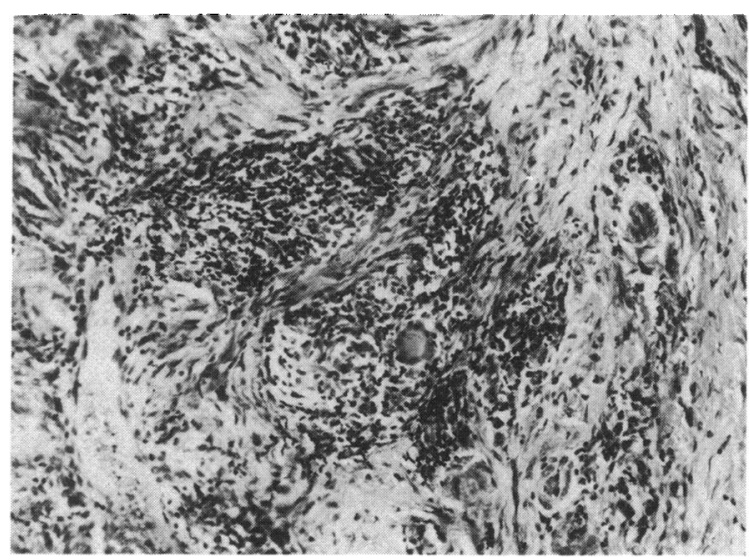

Figure 2 Tuberculous granuloma with lymphocytes, histiocytes, and foreign body giant cells ( $H$ and $E, \times 100)$. 
venereal disease research laboratory (VDRL) test was non-reactive. Chest and abdomen radiographs were normal.

Haematoxylin-eosin stained tissue sections from the lesion revealed the formation of multiple tuberculoid granulomas. They were located in the midand lower dermis. Each granuloma was formed by epithelioid cells located in the centre and lymphocytes at the periphery; Langhan's giant cells were present intermixed with epithelioid cells. However, no caseation necrosis was seen (fig 2), and acid-fast bacilli were not demonstrated by Ziehl-Neelsen stain. Mycobacterium tuberculosis was not recovered on culture, so further investigations such as niacin test could not be performed to rule out atypical mycobacteriosis.

Oral short course intensive antitubercular therapy for a period of 6 months comprising INAH $300 \mathrm{mg}$, rifampicin $600 \mathrm{mg}$, ethambutol $800 \mathrm{mg}$ and pyrazinamide $1500 \mathrm{mg}$ daily for an initial 2 months, followed by INAH and rifampicin for a further period of 4 months. ${ }^{23}$ The response to treatment was excellent and was marked by considerable decrease in induration and scaling of the plaque in the course of 4 months.

\section{Discussion}

Secondary cutaneous tuberculosis is now conceived to manifest either as reinfection or reactivation tuberculosis. ${ }^{1}$ It forms a continuous spectrum with lupus vulgaris a high cell-mediated response and tuberculosis cutis orifacialis representing impaired cell-mediated immunity. These polar forms are divided by tuberculosis verrucosa cutis and scrofuloderma, a situation akin to systemic tuberculosis. ${ }^{4}$

Its presentation as a mirror image lesion in the perineum appears to be unique and suggests an autoinoculation and therefore has the potential of infectivity to the other areas of the body. This is an intriguing observation for the acid-fast bacilli are usually difficult to demonstrate either in smear or tissue sections or recovered on culture. ${ }^{5}$ Such lesions should be differentiated from condylomata lata, a relatively common condition at this site. Shorter duration, morphological characteristics of the lesion, presence of Treponema pallidum on dark-ground illumination, reactive blood serology, and histopathology may confirm the diagnosis. Sarcoidosis, although an uncommon condition in the Indian subcontinent, should be considered on the basis of grains of sand studding the plaque, depressed or absent delayed hypersensitivity to tuberculin, a naked granuloma, and absence of therapeutic response to specific antitubercular treatment.

The importance of tuberculosis per se has increased with the advent of HIV infection. ${ }^{6-8}$ As the HIV infected population continues to increase, the incidence of cutaneous tuberculosis is also likely to rise. Furthermore, it is emphasised that short course intensive antitubercular chemotherapy is of immense value, and it not only helps in confirming the diagnosis, but also reduces morbidity and possibility of transmission of the disease.

Address for correspondence to Prof V N Sehgal, MD, FNASc, FAMS, A/6 Panchwati, Opp Azadpur Subzi Mandi, Delhi-110 033, India.

1 Sehgal VN, Wagh SA. Cutaneous tuberculosis current concepts. Int J Dermatol 1990;29:237-52.

2 Dutt AK, Moers D, Stead WW. Short course chemotherapy for extrapulmonary tuberculosis. Nine years experience. Ann Intern Med 1981;104:7-12.

3 Grosset JH. Present status of chemotherapy for tuberculosis. Rev Inf Dis 1989;2(Supp 2):347-52.

4 Ridley DS, Ridley MJ. Rationale for the histological spectrum of tuberculosis. A basis for classification. Pathology 1986;19: 186-92.

5 Sehgal VN, Jain MK, Srivastava G. Changing pattern of cutaneous tuberculosis. Int J Dermatol 1989;28:231-6. .

6 Sederam G, McDonald RJ, Maniatis T, et al. Tuberculosis as a manifestation of acquired immunodeficiency syndrome (AIDS). JAMA 1986;256:362-6.

7 Anonymous. Centers for Disease Control. Tuberculosis and acquired immunodeficiency syndrome. $M M W R$ 1986;34: 299-307.

8 Anonymous. Centers for Disease Control. Tuberculosis and acquired immunodeficiency syndrome. $M M W R$ 1986;35: 74-76.

Accepted for publication 9 April 1991 